\begin{tabular}{|c|l|}
\hline Title & Scale independent model of gravitational settling of particulate suspension in a fractal channel \\
\hline Author(s) & Kurose, Y udai; I shizawa, Kanami; Soriano, Ma Rebecca; Harada, Shusaku \\
\hline Citation & $\begin{array}{l}\text { Chemical Engineering Science, 199, 231-239 } \\
\text { https://doi.org/10.1016/.ces.2018.12.049 }\end{array}$ \\
\hline Issue Date & 2019-05-18 \\
\hline Doc URL & http://hdl.handle.net/2115/81398 \\
\hline Rights & $\begin{array}{l}\text { O2019. This manuscript version is made available under the CC-BY-NC-ND 4.0 license } \\
\text { http://creativecommons.org/icenses/by-nc-nd/4.0/ }\end{array}$ \\
\hline Rights(URL) & http://creativecommons.org/icenses/by-nc-nd/4.0/ \\
\hline Type & article (author version) \\
\hline hile Information & harada.pdf \\
\hline
\end{tabular}

Instructions for use 


\title{
Scale-Independent Model of Gravitational Dispersion of Particulate Suspension in a Fractal Channel
}

\author{
Yudai Kurose $^{\dagger}$, Kanami Ishizawa ${ }^{\dagger}$, Ma. Rebecca Soriano ${ }^{\dagger}$ and Shusaku Harada ${ }^{\dagger}$
}

$\dagger$ Division of Sustainable Resources Engineering, Faculty of Engineering, Hokkaido Univ. N13W8, Sapporo, Hokkaido 060-8628, Japan

Corresponding Author : Shusaku Harada

Address : Division of Sustainable Resources Engineering,

Faculty of Engineering, Hokkaido University

N13W8, Sapporo, Hokkaido 060-8628, Japan

Tel/Fax : +81-11-706-6310

E-mail : harada@eng.hokudai.ac.jp 


\begin{abstract}
Gravitational settling of solid particles into a fractal-shaped channel was investigated experimentally and theoretically. Previous studies have reported that the settling behavior of particles in liquid-filled channels depends strongly on particle properties and suspension conditions. At small particle size and high concentration, particles settle collectively like an immiscible fluid with respect to the surrounding one. In this study, we examined the gravitational dispersion behavior of solid particles in a three-dimensional fractal-shaped channel under various collective conditions. The experimental results showed that the settling behavior varies with the collectivity of suspended particles. In the case of high collectivity conditions, settling velocity is enhanced by a density-driven instability, which depends not only on the physical properties of the particles and fluid but also on channel geometry. We developed a model of temporal change in the volumetric occupancy ratio in a suspended region. This model describes the invasion of particles into a fractal channel. Our model comprises only the fractal characteristics of the channel, such as a homothetic ratio and a bifurcation number. Consequently, This model could provide a rough prediction of the gravity-induced invasion behavior of particles into any fractal-shaped channel.
\end{abstract}

Keywords : particulate suspension; gravitational settling; dispersion; complex channel; fractal characteristics 


\section{Introduction}

Gravitational dispersion of particulate materials in liquid-filled complex-shaped channels plays an important role in many engineering and natural processes, such as contaminant dispersion in water-saturated soil, filtration of muddy water and mass transport in porous media. However, quantitative prediction of the settling process of particles in complex channels still remains a challenge. This is because the settling behavior is affected not only by the physical properties of the suspended particles and carrier fluid, but also by geometric properties of flow channels such as channel length, cross-sectional shape, inclination and bifurcation. The detailed mechanism of gravitational dispersion in these channels has not yet been adequately understood.

Indeed, the settling behavior of solid particles in fluid is a classic fundamental issue of fluid mechanics. As is well-known, the settling velocity of an isolated particle in an infinite static fluid is determined by the combination of the gravitational force, buoyant force and drag force. For example, settling velocity at a low Reynolds number is known as Stokes settling velocity $U_{S t}=\left(\rho_{p}-\rho_{f}\right) d_{p}^{2} g / 18 \mu\left(\rho_{p}\right.$ : particle density, $\rho_{f}$ : fluid density, $d_{p}$ : particle diameter, $g$ : gravitational acceleration and $\mu$ : viscosity). However, the settling of many particles in fluid is extremely complicated due to the hydrodynamic interaction between particles that affects their settling motion $[1-3]$. As an example, the settling of particle clouds in a quiescent fluid shows various strange behaviors depending on the Reynolds number condition [4-6].

The settling of a stratified suspension in a simple flow channel also shows complicated behaviors depending on the suspension conditions [7-11]. At large particle diameter and low concentration, suspended particles settle individually (particle-like settling) at terminal velocity. On the other hand, at small particle diameter and high concentration, the particles settle collectively as a continuous fluid (fluid-like settling) [9]. In this case, the settling motion is governed by interface instability (Rayleigh-Taylor instability) at the concentration interface $[12,13]$. This phenomenon, which is also known as the Boycott effect, enhances settling velocity by means of the convection caused by the instability [14]. 
Complex-shaped channels with bifurcation or with variable cross-sectional area can be found in many natural systems. The settling behavior of suspended particles in such a complex channel is expected to be more complicated than in a simple channel. This study investigates how the particles settle gravitationally in a liquid-filled complex-shaped channel. Experiments were conducted using a multi-branched channel with fractal characteristics, wherein the geometric characteristics of the branches (e.g., ratio of channel lengths, homothetic ratio, bifurcation number) are constant throughout the channel [15]. In particular, we focus on the effect of the collective behavior of suspended particles settling in a fractal channel.

Previous work shows that the collectivity of settling particles can be expressed by a dimensionless parameter that is a function of particle diameter, concentration and channel geometry [9]. This dimensionless parameter determines whether the suspended particles behave individually (particle-like) or collectively (fluid-like). In this study, we examine the settling behaviors of suspended particles in the fractal channel under various conditions of the collectivity, i.e., particle-like or fluid-like conditions. Settling behaviors of particles are recorded by a multi-camera system and the settling velocity and the occupancy ratio of the suspended region are calculated by image analysis. Additionally, we develop a scale-independent model of temporal change in the occupancy ratio of suspended particles. This model comprises only the fractal characteristics of the channel for specific conditions of particulate suspension. Therefore the proposed model is expected to predict the gravitational dispersion behavior of suspended particles in any fractal-shaped channel regardless of lengthscale.

\section{Experimental Setup}

Experiments were conducted using a three-dimensional fractal-shaped channel. Figure 1 shows a schematic diagram of the experimental channel. The channel was composed of multi-branched channels of various sizes. These channels were categorized into four levels according to channel size. The lengths of each channel $(T \times D \times L)$ are $50.0 \mathrm{~mm} \times 25.0 \mathrm{~mm} \times 90.0 \mathrm{~mm}$ (level 1), 
$31.0 \mathrm{~mm} \times 15.5 \mathrm{~mm} \times 55.8 \mathrm{~mm}($ level 2), $19.2 \mathrm{~mm} \times 9.6 \mathrm{~mm} \times 34.6 \mathrm{~mm}($ level 3) and $11.9 \mathrm{~mm} \times 6.0 \mathrm{~mm}$ $\times 21.5 \mathrm{~mm}$ (level 4), respectively. Each level of the channel had the same geometric properties, i.e., a cross-sectional aspect ratio $\Gamma_{T}=T / D=2$, a longitudinal aspect ratio $\Gamma_{L}=L / D=3.6$, a homothetic ratio $\psi=0.62$ and a number of branches $N=4$. These channel dimensions were carved into a $200 \mathrm{~mm} \times 200 \mathrm{~mm} \times 180 \mathrm{~mm}$ transparent acrylic block. The whole fractal channel system was composed of interlocking blocks piled in a transparent box. The outer box had a container in the upper part for the suspension reservoir, with a thin horizontal slit between the container and the channel.

The procedure for preparation of the experiment was as follows. Pure silicone oil (density $\rho_{f}=972 \mathrm{~kg} / \mathrm{m}^{3}$, viscosity $\left.\mu=1.94 \mathrm{~Pa} \cdot \mathrm{s}\right)$ was first poured into the channel. Then a thin stainless blade (thickness $0.5 \mathrm{~mm}$ ) was put into the slit to separate the channel from the container. After air bleeding, the particulate suspension was poured into the upper container. Finally the blade was removed, and the settling behavior of particles was recorded by a multi-video camera system. The setup of the video camera system is described later.

Table 1 shows the properties of particulate suspension used in this study. The particles used in the experiments were mono-dispersed glass particles (density $\rho_{p}=2500 \mathrm{~kg} / \mathrm{m}^{3}$, diameter $d_{p}=$ $100 \mu \mathrm{m}$ ) or polystyrene particles (density $\rho_{p}=1050 \mathrm{~kg} / \mathrm{m}^{3}$, diameter $d_{p}=600 \mu \mathrm{m}$ or $800 \mu \mathrm{m}$ ). The fluid used in the suspension was the same as that used for filling the channel.

Experiments were conducted to examine how settling behavior changes as the collectivity of particulate suspension varies. As described in the Introduction, the previous study [9] found that a dimensionless parameter called "collectivity" $\zeta^{-1} \phi^{1 / 3}$, where $\zeta=d_{p} / D(D$ : short-side length of cross-section of rectangular channel), determines the collectivity in a Hele-Shaw channel. In this study, we define $\zeta=d_{p} / D_{1}\left(D_{1}\right.$ : short-side length of the cross-section of level 1 channel) and calculate the collectivity as $\zeta^{-1} \phi^{1 / 3}$.

As shown in Table 1, we attempted experiments with various collectivity conditions, i.e., particle-like condition, middle condition and fluid-like condition (conditions I to III). We used 
polystyrene particles $\left(d_{p}=800 \mu \mathrm{m}\right)$ for condition I, polystyrene particles $\left(d_{p}=600 \mu \mathrm{m}\right)$ for condition II and glass particles $\left(d_{p}=100 \mu \mathrm{m}\right)$ for condition III. The concentration of suspension for each condition was $\phi$ is $0.00033,0.018$, and 0.05 , respectively. The collectivity $\zeta^{-1} \phi^{1 / 3}$ calculated from the particle diameter and the concentration was 2.16 (particle-like), 11.0 (middle) and 92.1 (fluid-like) respectively.

As previously described, one of the purposes of this study is to develop a model to predict the settling behavior of particulate suspension under fluid-like (high collectivity) conditions. In order to establish the model, additional experiments were conducted with three different collectivity conditions. We used glass particle suspensions for $\phi$ is $0.03,0.05$ and 0.08 (conditions III through V). The corresponding collectivity values for these conditions were 77.7, 92.1 and 107.2, respectively. These values denote experimental conditions of adequately fluid-like condition.

Several video cameras were used to observe settling behavior in each branched-channel from level 1 to level 4. Most of the cameras were aimed perpendicular to the long or short side of a channel, in order to observe the settling behavior in each level of the channel. Other cameras were used for the front view and the bottom view, in order to observe the overall motion of suspension in the fractal channel. The occupancy volume and the invasion velocity of suspension in each level were quantified through image analysis from successive pictures in each level of channel. For calibration of the image analysis, an image with scale was initially taken in each level of channel.

\section{Results and Discussion}

\section{Settling Behavior of Particulate Suspension in Fractal Channel}

In order to know whether settling behavior of suspended particles varies with the collectivity, experiments were conducted under three typical conditions, i.e., particle-like (condition I), middle (condition II) and fluid-like (condition III). Figure 2 shows the observational results of settling behaviors of particulate suspensions with different collectivity. Note that the settling times are 
indicated in minutes for (a) particle-like and (b) middle conditions, while in seconds for (c) fluid-like condition.

As shown in Fig.2(a), suspended particles settled individually relative to the surrounding fluid under the particle-like condition. In the level 1 channel, particles settled almost vertically while spreading throughout the channel. Under this condition, particle trajectories did not change even after passing through a channel bifurcation. Consequently, particle accumulated near the inclined wall of the channel and then settled down into the branches near the center.

On the other hand, the settling of suspended particles with high collectivity showed behaviors different from those with low collectivity. As can be seen in Fig.2(b), the suspended particles settled near the center of the channel forming a finger-like blob in level 1 under the middle condition. After passing the bifurcation, the particles moved laterally and spreaded into many branches. In the case of the highest collectivity shown in Fig.2(c), the particulate suspension settled collectively at the center of the channel. Under this condition, the suspended particles behaved as an immiscible fluid with surrounding one $[9,10]$. Consequently, volume exchange between the suspension and the lower pure fluid occured in the channel. The settling velocity of the suspended particles with high collectivity was enhanced by Rayleigh-Taylor instability at the concentration interface, which is the apparent interface between particulate suspension and pure fluid [9]. Our previous study reported that if the cross-sectional aspect ratio of channel $\Gamma_{T}$ was less than 5 , the suspended region formed one finger at the center of the channel and the size of finger was approximately a half of the long side length of channel cross-section [10]. The channel aspect ratio in the present experiment was $\Gamma_{T}=2$, and it can be observed from Fig.2(c) that the finger-like blob occupies around half of the channel.

Figure 2(c) also shows a bottom view of the settling behavior of particulate suspension under a fluid-like condition. From experimental observation, we found that suspended particles invaded into all branches almost symmetrically and that finger size shrinked with decreasing channel size. 
These results indicate that the suspension behaved as an immiscible fluid with respect to the surrounding fluid and the settling behavior is dominated by volume exchange effects. From these experimental observations, we found that the invasion behavior of particulate suspension into the fractal channel was crucially complicated under a fluid-like condition.

Figure 3 indicates the settling velocity of particles in the channel of level $1 U_{1}$ for a range of collectivity values. Settling velocity is normalized with the Stokes settling velocity $U_{S t}$ according to particle conditions given in Table 1. As described in the previous study, the settling velocity greatly depended on the collectivity [9]. In the case of a low collectivity condition, the settling velocity was almost identical to the terminal velocity of an isolated particle $U_{S t}$. On the other hand, the settling velocity in the case of a high collectivity condition was several hundred times larger than the Stokes settling velocity since it was strongly enhanced by the large-scale convection caused by the collective settling.

\section{Basic Concept of Dispersion Model for Collective Settling}

As explained in Introduction, one of the purposes in this study is to develop a model of gravitational dispersion of particulate suspension with high collectivity in a fractal channel. A versatile model should be able to predict the invasion rate of the particulate suspension into branched channels with arbitrary fractal characteristics. For this reason, the dispersion model should be a function of fractal properties of the channel. By establishing such a model, we can predict the invasion behavior of the suspension into any fractal-shaped channel, even if particle behavior cannot be observed from outside. Hereafter, we develop a mathematical model for the temporal change in occupancy rate (invasion rate) of the particulate suspension in each branch of the fractal channel from the experimental results under fluid-like conditions (conditions III to V in Table 1).

\section{Cross-Sectional Occupancy Ratio for Each Level}

As shown in Fig.2(c), settling suspension with high collectivity forms a finger-like blob at the 
center of each channel. We also observed that the size of the blob depends on the channel width. In order to model the occupancy rate of the suspension in each channel, the cross-sectional ratio of the suspended region was estimated through image analysis.

Figure 4 indicates the cross-sectional occupancy ratio of the suspended region to the channel at each level for concentrations $\phi=0.03,0.05$ and 0.08 (conditions III, IV and V), respectively. As shown in the figure, the cross-sectional occupancy ratio was about 0.4 regardless of the channel level. These observational results show that the long-side length of the finger was nearly half the channel length. On the other hand, the short-side length of the finger was about $80 \%$ of the channel length. This was because an upward flow was generated near the wall acting as a boundary layer. Our previous study indicated that the finger width was almost half the long-side channel length in a vertical rectangular channel with the aspect ratio $\Gamma_{T}<5$ [10]. The finger width shown in the present experiment almost coincided with these previous results even in the inclined channels. In high collectivity cases, the suspension settled in an immiscible fashion with the lower fluid. The reason why the cross-sectional occupancy ratio was always less than 0.5 was that the volume exchange between the upper suspension and the lower fluid occured somewhat asymmetrically. Based on the results indicated in Fig.4, the cross-sectional occupancy ratio was set to 0.4 for the dispersion model in a fractal channel regardless of channel level.

\section{Settling Velocity at Each Level of Channel}

The previous study showed that the settling velocity of suspended particles with high collectivity depended on the cross-sectional aspect ratio of the channel [10]. Figure 5 shows the previous results regarding the settling velocity of particulate suspension in a rectangular channel with various crosssectional geometries. As seen in the figure, the settling pattern of the suspension divided into certain regions according to the aspect ratio of the channel. If the long side of the channel was adequately large, many fingers with similar sizes grew up in the channel (infinite region). On the 
other hand, if the cross-sectional aspect ratio of the channel was less than 5 , one finger developed at the center of the channel with the settling velocity depending on the channel aspect ratio (linear region). In this section, we develop a model for the collective settling velocity of suspended particles in a fractal channel.

Based on the results shown in Fig.5, the velocity of a finger in a rectangular channel can be expressed as a function of the channel cross-sectional aspect ratio as follows;

$$
U= \begin{cases}0.275(T / D) U^{\infty} & \text { for } T / D<5 \\ U^{\infty} & \text { for } T / D \geq 5\end{cases}
$$

where $D$ is the short-side length and $T$ is the long-side length of the rectangular channel. $U^{\infty}$ is the settling velocity in a Hele-Shaw channel (large aspect ratio). From the previous study, $U^{\infty}$ is described as a function of collectivity in the following way $[9,10]$;

$$
U^{\infty}=0.107\left(\zeta^{-1} \phi^{1 / 3}\right)^{2} U_{S t}
$$

where $U_{S t}$ is the Stokes settling velocity of constituent particle and $\zeta$ is the particle diameter normalized by the short-side length of channel such that $\zeta=d_{p} / D$.

From these previous results, we derive the settling velocity of the suspension for each level of a fractal channel. As mentioned above, all channel levels of in our experiment have the same fractal characteristics and the cross-sectional aspect ratio $\Gamma_{T}=T_{1} / D_{1}=T_{2} / D_{2}=\cdots=T_{i} / D_{i}=2$. According to Eq.(1), the settling velocity at level $i$ should be proportional to the aspect ratio as follows;

$$
U_{i}=0.275 \frac{T_{i}}{D_{i}} U_{i}^{\infty}=0.275 \Gamma_{T} U_{i}^{\infty}
$$

where $U_{i}^{\infty}$ is the settling velocity in a Hele-Shaw channel with short-side length $D_{i}$ and is derived from Eq.(2) as follows;

$$
U_{i}^{\infty}=0.107\left(\zeta_{i}^{-1} \phi^{1 / 3}\right)^{2} U_{S t}=0.107\left(\frac{D_{i} \phi^{1 / 3}}{d_{p}}\right)^{2} U_{S t}
$$


where $\zeta_{i}=d_{p} / D_{i}$. If we rewrite Eq.(4) using the homothetic ratio in the fractal channel $\psi=$ $D_{2} / D_{1}=D_{3} / D_{2}=\cdots=D_{i} / D_{i-1}$, the following relation is obtained.

$$
U_{i}^{\infty}=0.107\left(\frac{D_{1} \phi^{1 / 3}}{d_{p}}\right)^{2}\left(\frac{D_{i}}{D_{1}}\right)^{2} U_{S t}=0.107\left(\frac{D_{1} \phi^{1 / 3}}{d_{p}}\right)^{2} \psi^{2(i-1)} U_{S t}
$$

By substituting Eq.(5) into Eq.(3), the settling velocity in level $i$ can be derived as follows;

$$
U_{i}=k \Gamma_{T} \psi^{2(i-1)} U_{S t}
$$

where $k=0.0294\left(\zeta^{-1} \phi^{1 / 3}\right)^{2}$. In this expression, we redefine the normalized particle diameter in the fractal channel as $\zeta=d_{p} / D_{1} . k$ is a constant determined from the properties of suspended particles and the fractal characteristics of the channel. As can be seen in Eq.(6), the collective settling velocity of suspended particles for each level of the fractal channel is represented solely by the fractal characteristics of the channel $\left(\Gamma_{T}\right.$ and $\left.\psi\right)$ under a given condition of particulate suspension.

Figure 6 shows the settling velocity for each channel level obtained from the experiments and the prediction from Eq.(6) for glass particle suspension with concentrations $\phi=0.03,0.05$ and 0.08 (conditions III thtough V), respectively. The results from Eq.(6) almost predict the settling velocities for each level, even though Eq.(6) assumes vertical settling. However, the predicted results underestimate settling velocity after the first bifurcation, particularly for level 2 . These underestimates are mainly caused by the mass flux imbalance of the suspension at the bifurcation point. Model corrections that consider mass balance are explained later. As mentioned in the Introduction, the main goal of this study is to develop the predictive model for the invasion behavior of particulate suspension into an arbitrarily complex fractal channel whose contents are invisible from outside. In this context, a model constructed solely from the fractal characteristics of the channel constitutes a significant advance, even if it gives only a rough estimate.

\section{Modeling of Fractal Channel Volume}

In this section, we generalize the volume of a fractal channel. The total volume of the channel 
$V_{\text {all }}$ is calculated by summing the channel volume at each channel as follows;

$$
V_{\text {all }}=L_{1} T_{1} D_{1}+N L_{2} T_{2} D_{2} \cdots+N^{i-1} L_{i} T_{i} D_{i}+\cdots
$$

where $L_{i}, T_{i}$ and $D_{i}$ are the channel lengths for level $i$ and $N$ is the number of branches. By using the homothetic ratio $\psi=L_{i} / L_{i-1}=T_{i} / T_{i-1}=D_{i} / D_{i-1}$, Eq.(7) can be expressed as follows;

$$
V_{\text {all }}=L_{1} T_{1} D_{1}\left[1+N \psi^{3}+\left(N \psi^{3}\right)^{2}+\cdots+\left(N \psi^{3}\right)^{i-1}+\cdots\right]
$$

Using the equation describing the fractal channel length $\left(L_{1}=\Gamma_{L} D_{1}\right.$ and $\left.T_{1}=\Gamma_{T} D_{1}\right)$ and applying the sum of the infinite geometric series, we can rewrite Eq.(8) in the following way.

$$
V_{\text {all }}=\frac{\Gamma_{L} \Gamma_{T} D_{1}^{3}}{1-N \psi^{3}}
$$

Note that Eq.(9) is composed of the fractal characteristics of the channel except for $D_{1}$.

\section{Scale-Independent Model of Occupancy Ratio}

Based on the results in the previous sections, we develop a predictive model for the temporal change in occupancy ratio of suspended particles in a fractal channel. Figure 7 shows a conceptual diagram of the model for the invasion of the suspension into a fractal channel. As indicated in the figure, we model a temporal change of occupancy ratio for each channel level. The total occupancy ratio of the suspension is calculated by summing up the results in overall channels. To model the invasion behavior of the suspension into each channel, we use the sigmoid function as follows;

$$
f(t)=\frac{1}{1+e^{-\alpha t}}
$$

where $t[\mathrm{~s}]$ is time and $\alpha\left[\mathrm{s}^{-1}\right]$ is a parameter. In general, the sigmoid function is close to constant values for $t=-\infty$ and $t=\infty$, and the gradient at the center of the slope is $\alpha / 4$. We model the invasion behavior into a fractal channel by superposing the sigmoid functions for all levels. As shown in Fig.7, the maximum gradient of the sigmoid function corresponds to the inverse of the 
travel time of suspension for each channel level $U_{i} / L_{i}$. Therefore the parameter of the sigmoid function should be $\alpha=4 U_{i} / L_{i}$.

First, we model the temporal change in suspension volume for each channel level $V_{i}$. As shown in Fig. 4 , the cross-sectional occupancy area is $40 \%$ of $T_{i} D_{i}$ regardless of channel size. Consequently the invasion behavior of suspended particles into the channel at level $i$ can be expressed as follows;

$$
\frac{d V_{i}}{d t}=\frac{2}{5} T_{i} D_{i} U_{i}
$$

Equation (11) is expressed approximately as a continuous function of time using sigmoid function.

Since the maximum suspension volume for each level is also $40 \%$ of the channel volume $L_{i} T_{i} D_{i}$, the invasion behavior of the suspension into the channel for level $i$ is modeled by the sigmoid function as follows;

$$
V_{i}(t)=\frac{\frac{2}{5} L_{i} T_{i} D_{i}}{1+\exp \left[-\alpha\left(t-t_{i}\right)\right]}=\frac{\frac{2}{5} \psi^{3(i-1)} \Gamma_{L} \Gamma_{T} D_{1}^{3}}{1+\exp \left[-\alpha\left(t-t_{i}\right)\right]}
$$

where we use the relation for fractal characteristics $L_{i}=\Gamma_{L} D_{i}, T_{i}=\Gamma_{T} D_{i}$ and $\psi=L_{i} / L_{i-1}=$ $T_{i} / T_{i-1}=D_{i} / D_{i-1}$

In Eq.(12), $t_{i}$ is the time at which suspended particles reach halfway down the each channel length for level $i$ (see Fig.7) and $\alpha$ is the parameter of the sigmoid function. As mentioned above, $\alpha$ is related to the settling velocity and $\alpha=4 U_{i} / L_{i}$. From Eq.(6), we can obtain the parameter $\alpha$ in the following way.

$$
\alpha=\frac{4 U_{i}}{L_{i}}=\frac{4 k \Gamma_{T} \psi^{2(i-1)} U_{S t}}{\Gamma_{L} \psi^{i-1} D_{1}}=\frac{4 k \Gamma_{T} \psi^{i-1} U_{S t}}{\Gamma_{L} D_{1}}
$$

On the other hand, taking into account the travel time for the suspension in previous levels, $t_{i}$ can be expressed as follows;

$$
t_{i}=\left(\frac{L_{1}}{U_{1}}+\frac{L_{2}}{U_{2}}+\cdots+\frac{L_{i-1}}{U_{i-1}}\right)+\frac{L_{i}}{2 U_{i}}
$$

In the same way as Eq.(13), we can express $t_{i}$ in terms of the fractal characteristics as follows;

$$
\begin{aligned}
t_{i} & =\frac{\Gamma_{L} D_{1}}{k \Gamma_{T} U_{S t}}\left(1+\frac{1}{\psi}+\cdots+\frac{1}{\psi^{i-2}}\right)+\frac{\Gamma_{L} D_{1}}{2 k \Gamma_{T} U_{S t} \psi^{i-1}} \\
& =\frac{\Gamma_{L} D_{1}}{2 k \Gamma_{T} U_{S t}}\left(2 \frac{\psi^{i-2}+\psi^{i-3}+\cdots+1}{\psi^{i-2}}+\frac{1}{\psi^{i-1}}\right)
\end{aligned}
$$


The numerator of the first term in Eq.(15) is regarded as a finite geometric series which can be written as $\left(1-\psi^{i-1}\right) /(1-\psi)$. Finally we obtain $t_{i}$ in the following way.

$$
t_{i}=\frac{\Gamma_{L} D_{1}}{2 k \Gamma_{T} U_{S t}}\left(\frac{\psi-2 \psi^{i}+1}{\psi^{i-1}(1-\psi)}\right)
$$

The temporal change in occupancy ratio of suspended particles in a fractal channel $\sigma_{F}(t)$ is expressed as the total occupancy volume of the suspension divided by the volume of the fractal channel $V_{\text {all }}$ as follows;

$$
\sigma_{F}(t)=\frac{1}{V_{\text {all }}} \sum_{i} N^{i-1} V_{i}(t)
$$

By substituting Eqs.(9), (13) and (16) into Eq.(17), we establish the temporal model for occupancy ratio of the suspension in a fractal channel;

$$
\sigma_{F}\left(t^{*}\right)=\frac{2}{5}\left(1-N \psi^{3}\right) \sum_{i} \frac{\left(N \psi^{3}\right)^{i-1}}{1+\exp \left(-4 \frac{k \Gamma_{T} \psi^{i-1}}{\Gamma_{L}} t^{*}+2 \frac{\psi-2 \psi^{i}+1}{1-\psi}\right)}
$$

where $t^{*}$ indicates the normalized time $t U_{S t} / D_{1}$. The most remarkable feature of the model described in Eq.(18) is that the temporal change in occupancy ratio is expressed solely in terms of the fractal characteristics of the channel $\left(\Gamma_{T}, \Gamma_{L}, \psi\right.$ and $\left.N\right)$. This means that the occupancy ratio does not depend on any lengthscales. Thus, this model can predict the invasion behavior of particulate suspension from the fractal nature of channels alone. However, as described later, the model expressed by Eq.(18) does not predict the suspension behavior well in some cases (see Fig.8). Therefore some correction of the model is required as explained in the next section.

\section{Model Correction for Accumulation Effects}

The model given by Eq.(18) is based on the superposition of occupancy volumes over all levels. Consequently, this model does not satisfy the mass conservation during particle settling. Therefore we further develop the model to take into account the accumulation of particles at bifurcations. We assume that accumulation occur due to the difference between inlet and outlet mass flux of 
particles. The accumulation rate at the bifurcation point between level $i$ and level $i+1$ can be expressed as follows;

$$
\frac{d V_{B i}}{d t}=\frac{2}{5} U_{i} T_{i} D_{i}-N \frac{2}{5} U_{i+1} T_{i+1} D_{i+1}
$$

where $V_{B i}$ is the accumulation volume at the bifurcation point. If we substitute Eq.(6) into Eq.(19) and express some quantities in terms of fractal characteristics, we can obtain the following equation.

$$
\frac{d V_{B i}}{d t}=\frac{2}{5} k U_{S t} \Gamma_{T} \psi^{2(i-1)} T_{i} D_{i}\left(1-N \psi^{4}\right)
$$

The temporal change in accumulation volume is also modeled by the sigmoid function given in Eq.(10). Considering that the maximum accumulation volume (vacant volume) at level $i$ is $60 \%$ of the channel volume $L_{i} T_{i} D_{i}$, the accumulation volume is expressed as follows.

$$
V_{B i}(t)=\frac{\frac{3}{5} L_{i} T_{i} D_{i}}{1+\exp \left[-\alpha_{B}\left(t-t_{B i}\right)\right]}
$$

The parameter of the sigmoid function $\alpha_{B}$ can be expressed in a way similar to Eq.(13) as;

$$
\alpha_{B}=4 \frac{\frac{2}{5} k U_{S t} \Gamma_{T} \psi^{2(i-1)} T_{i} D_{i}\left(1-N \psi^{4}\right)}{\frac{3}{5} L_{i} T_{i} D_{i}}=\frac{8}{3} \frac{k U_{S t} \psi^{2(i-1)}\left(1-N \psi^{4}\right)}{L_{i}}
$$

On the other hand, $t_{B i}$ indicates the time at which suspended particles reach half the maximum accumulation volume at level $i$. It can be expressed in a way similar to Eq.(16);

$$
\begin{aligned}
t_{B i} & =\left(\frac{L_{1}}{U_{1}}+\frac{L_{2}}{U_{2}}+\cdots+\frac{L_{i}}{U_{i}}\right)+\frac{1}{2} \frac{\frac{3}{5} L_{i} T_{i} D_{i}}{\frac{2}{5} k U_{S t} \Gamma_{T} \psi^{2(i-1)} T_{i} D_{i}\left(1-N \psi^{4}\right)} \\
& =\frac{\Gamma_{L} D_{1}\left(1-\psi^{i}\right)}{k U_{S t} \Gamma_{T} \psi^{i-1}(1-\psi)}+\frac{3}{4} \frac{L_{i}}{k U_{S t} \Gamma_{T} \psi^{2(i-1)}\left(1-N \psi^{4}\right)}
\end{aligned}
$$

By substituting Eqs.(22) and (23) into Eq.(21), and dividing by the volume of the channel as in Eq.(18), the accumulation model of occupancy ratio is derived as follows;

$$
\sigma_{B}\left(t^{*}\right)=\frac{3}{5}\left(1-N \psi^{3}\right) \sum_{i} \frac{\left(N \psi^{3}\right)^{i-1}}{1+\exp \left[-\frac{8}{3}\left(1-N \psi^{4}\right)\left(\frac{k U_{S t} \Gamma_{T} \psi^{i-1}}{\Gamma_{L}} t^{*}-\frac{1-\psi^{i}}{1-\psi}\right)+2\right]}
$$

As indicated in Eq.(24), the accumulation model is also expressed by fractal characteristics alone. If we combine Eqs.(18) and (24), the final form of the model for occupancy ratio of suspended particles in a fractal channel is obtained as;

$$
\sigma\left(t^{*}\right)=\sigma_{F}\left(t^{*}\right)+\sigma_{B}\left(t^{*}\right)
$$


The occupancy model given in Eq.(25) is somewhat complicated. However, it is important that the model is independent of any lengthscale of a fractal channel.

Figure 8 shows the experimental results of the volumetric occupancy ratio of glass particle suspension in the fractal channel for $\phi=0.03,0.05$ and 0.08 (condition III through V in Table 1), respectively. The model results given in Eq.(18) (no accumulation effect) and Eq.(25) (considering accumulation effect) are also shown in the figure. In the model analysis, we calculate the occupancy ratio using the actual total volume of channels from level 1 to 4 instead of the infinite volume described in Eq.(9). It should be noted that some experimental data are missing in Fig.8. This is because image analysis cannot always capture the moment that the finger front just reaches at the bifurcation.

As shown in Fig.8, the model results closely match experimental results in all cases. It is found that the model that corrects for accumulation effects predicts the volumetric occupancy ratio after the first bifurcation quite well, while the predictions from the model with no accumulation effect drift gradually away from experimental results. As mentioned above, the occupancy model given in Eq.(25) consists of the superposition of invasion behaviors for all levels of the channel $\sigma_{F}$ and the correction for accumulation effect $\sigma_{B}$. The latter term involves all effects caused by the mass imbalance at each bifurcation point. This means that the proposed model gives a rough prediction of the invasion behavior of suspension in fractal channels. However, it should be emphasized again that the model proposed in this study is composed solely of the fractal characteristics of channels includes no lengthscale (scale-independent). Therefore the model is capable of a rough prediction of the gravitational dispersion behavior of particulate suspension in any fractal channel based on fractal characteristics alone.

\section{Conclusions}

Gravitational dispersion behavior of suspended particles in a liquid-filled fractal channel was investigated experimentally and theoretically. We examined differences in invasion behavior of the 
suspension into the channel under various suspension conditions. The dimensionless parameter "collectivity" characterizes the settling motion of suspended particles. In the case of low collectivity (large particle size and low concentration), the particles behave individually and settle almost vertically in a fractal channel. On the other hand, under high collectivity conditions, the suspended particles behave as an immiscible fluid and settle down much faster than an isolated particle due to density-driven instability. The suspension moves laterally and invades into many channels via volume replacement to the surrounding fluid.

The temporal change in occupancy ratio of the suspended region in a fractal channel was modelled under high collectivity conditions. The model developed in this study consists of the fractal characteristics of the channel only, therefore it is independent of any spatial lengthscale. The calculated predictions from the proposed model agree well with the experimental results. By using the model proposed in this study, it is possible to predict the gravitational dispersion behavior of particles in any fractal channel which is invisible from outside.

\section{Acknowledgment}

This study was supported by JSPS KAKENHI Grants No. 26420095. 


\section{References}

[1] R. H. Davis and A. Acrivos, Ann. Rev. Fluid Mech. 17 (1985) 91.

[2] J.- Z. Xue, E. Herbolzheimer, M. A. Rutgers, W. B. Russel and P. M. Chaikin, Phys. Rev. Lett. 69 (1992) 1715.

[3] E. Guazzelli, and J. Hinch, Ann. Rev. Fluid Mech. 43 (2011) 97.

[4] K. Adachi, S. Kiriyama and N. Yoshioka, Chem. Eng. Sci. 33 (1978) 115

[5] J. M. Nitsche and G. K. Batchelor, J. Fluid Mech. 340 (1997) 161.

[6] B. Metzger, M. Nicolas and E. Guazzeli, J. Fluid Mech. 580 (2007) 283.

[7] C. Völtz, M. Schröter, G. Iori, A. Betat, A. Lange, A. Engel and I. Rehberg, Phys. Rep. 337 (2000) 117.

[8] F. Blanchette and J. W. M. Bush, Phys. Fluids 17 (2005) 073302-1.

[9] S. Harada, T. Mitsui and T. Sato, Eur. Phys. J. E 35 (2012) 1.

[10] S. Harada, M. Kondo, M. Watanabe, K. Shiotani and T. Sato, Chem. Eng. Sci. 93 (2013) 307.

[11] Y. Yamamoto, F. Hisataka and S. Harada, Int. J. Multiphase Flow 73 (2015) 71.

[12] C. Völtz, W. Pesch and I. Rehberg, Phys. Rev. E 65 (2001) 011404.

[13] C. Völtz, Phys. Rev. E 68 (2003) 021408.

[14] E. Guyon, J.-P. Hulin, L. Petit and C. D. Mitescu, Physical Hydrodynamics, Oxford University Press (2001).

[15] S. Guha and K. Pradhan, Phys. Fluids 29 (2017) 063602. 


\section{Figure and Table list}

Table 1 Physical properties and suspension conditions of solid particles.

Fig.1 Schematic diagram of experimental channel.

Fig.2 Settling behavior of particulate suspensions in fractal channel with different collectivity

Fig.3 Comparison of settling velocity in level 1 channel under particle-like, middle and fluid-like conditions.

Fig.4 Cross-sectional occupancy ratio for each level of fractal channel

Fig.5 Settling velocity of particulate suspension in rectangular channel (reconstruction of Fig.8 in Harada et al. [10])

Fig.6 Settling velocity of glass particles for each level of fractal channel (open circles: experimental results, dotted lines: prediction from Eq.(6))

Fig.7 Conceptual diagram of model for invasion of particulate suspension into fractal channel

Fig.8 Comparison between experimental and model results for volumetric occupancy ratio 
Table 1 Physical properties and suspension conditions of solid particles.

\begin{tabular}{clccccc}
\hline & & diameter & density & Stokes velocity & concentration & collectivity \\
& & $d_{p}(\mu \mathrm{m})$ & $\rho_{p}\left(\mathrm{~kg} / \mathrm{m}^{3}\right)$ & $U_{S t}(\mathrm{~mm} / \mathrm{s})$ & $\phi$ & $\zeta^{-1} \phi^{1 / 3}$ \\
\hline (I) & polystyrene particle & 800 & 1050 & $1.40 \times 10^{-2}$ & 0.00033 & 2.16 \\
(II) & polystyrene particle & 600 & 1050 & $7.87 \times 10^{-3}$ & 0.018 & 11.0 \\
(III) & glass particle & 100 & 2500 & $4.28 \times 10^{-3}$ & 0.03 & 77.7 \\
(IV) & glass particle & 100 & 2500 & $4.28 \times 10^{-3}$ & 0.05 & 92.1 \\
(V) & glass particle & 100 & 2500 & $4.28 \times 10^{-3}$ & 0.08 & 107.2 \\
\hline
\end{tabular}




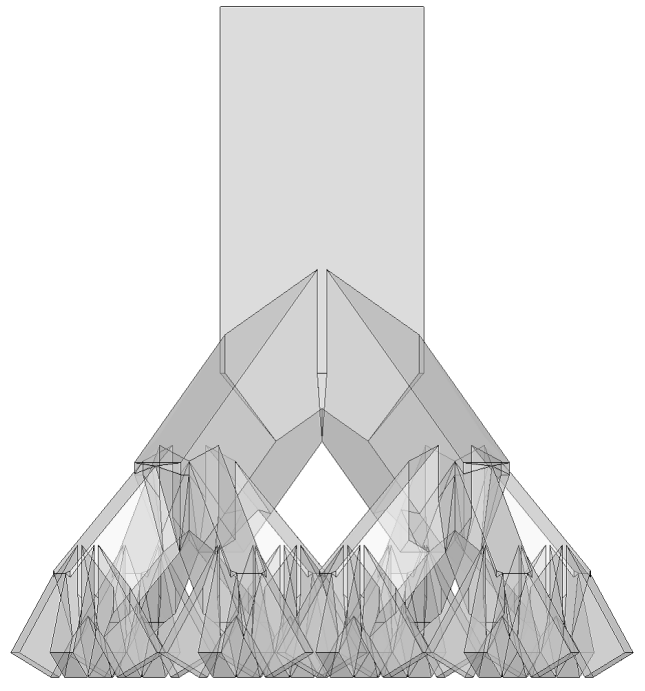

(a) front view

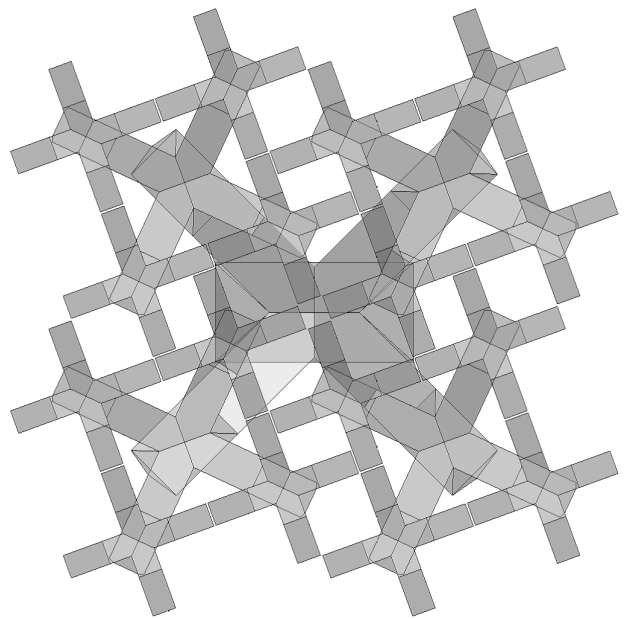

(b) bottom view

Fig.1 Schematic diagram of experimental channel. 


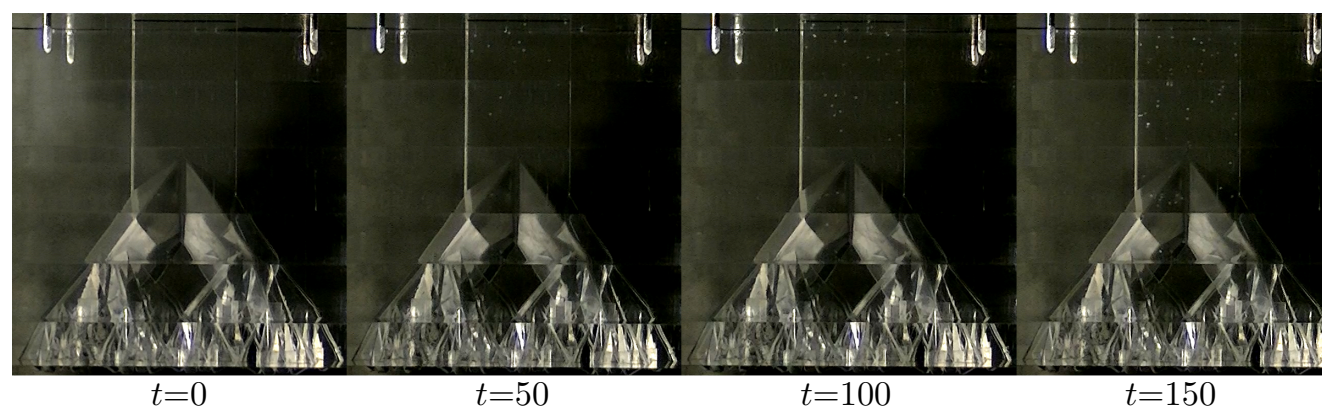

$t=200$ [min.]

(a) particle-like condition

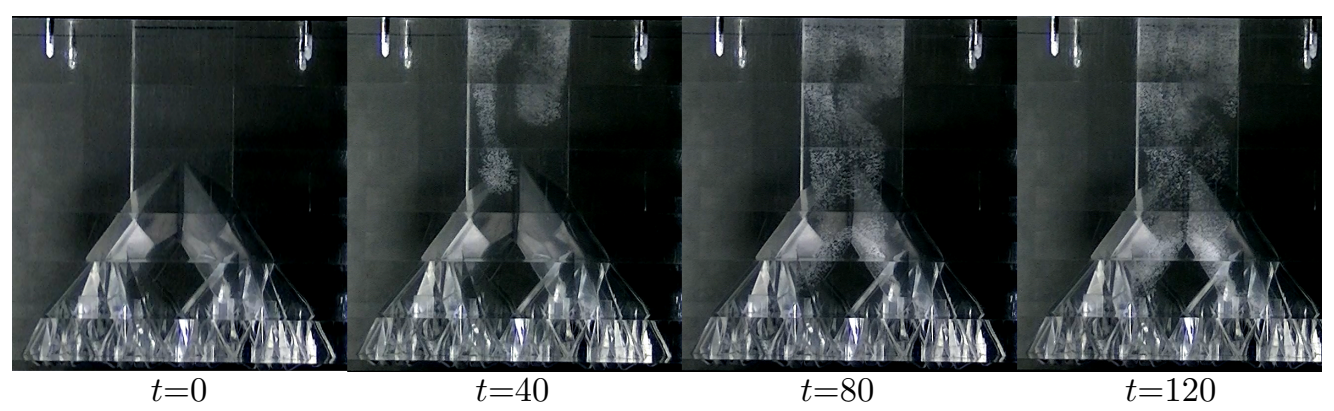

(b) middle condition

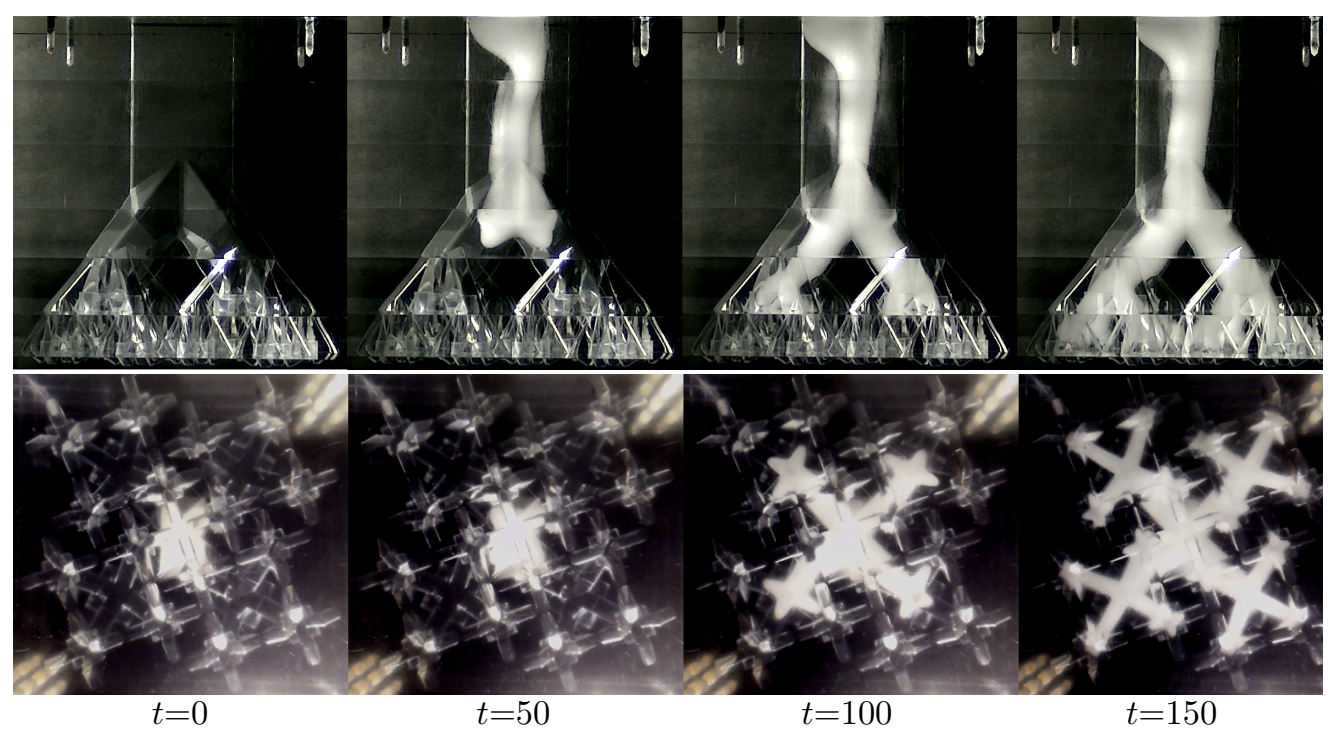

(c) fluid-like condition (upper: front view, lower: bottom view)

Fig.2 Settling behavior of particulate suspensions in fractal channel with different collectivity 


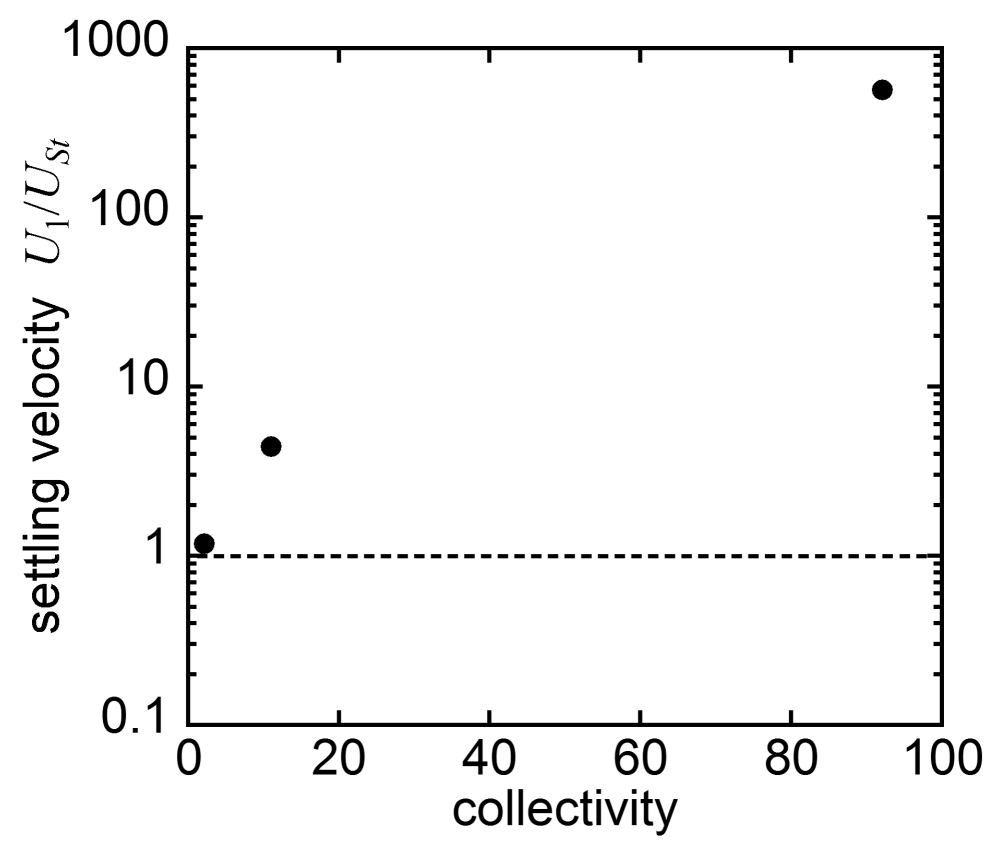

Fig.3 Comparison of settling velocity in level 1 channel under particle-like, middle and fluid-like conditions. 


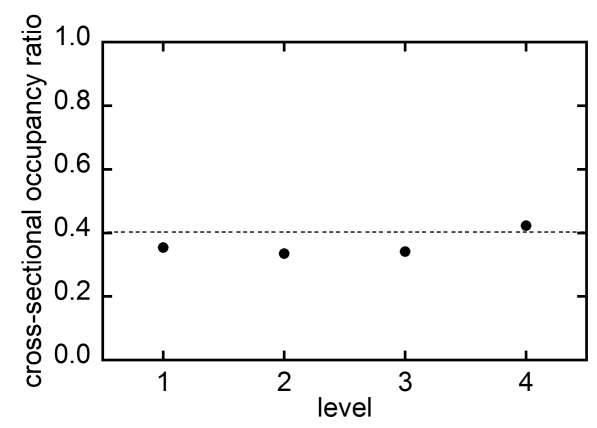

(a) $\phi=0.03$

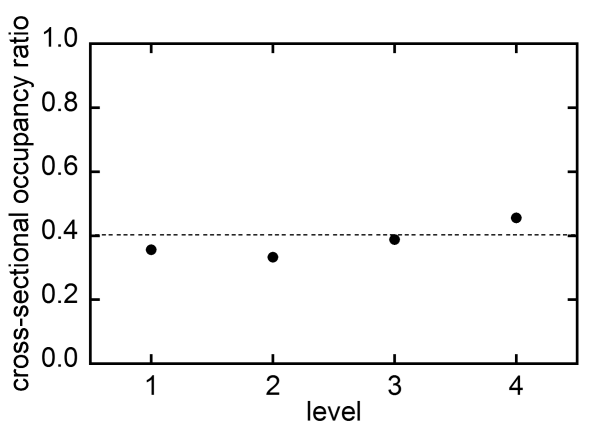

(b) $\phi=0.05$

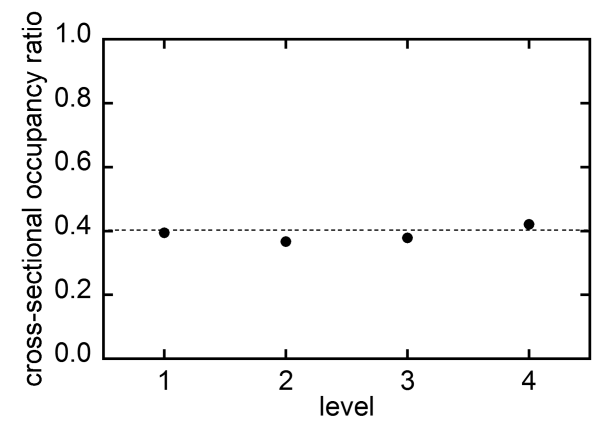

(c) $\phi=0.08$

Fig.4 Cross-sectional occupancy ratio for each level of fractal channel 


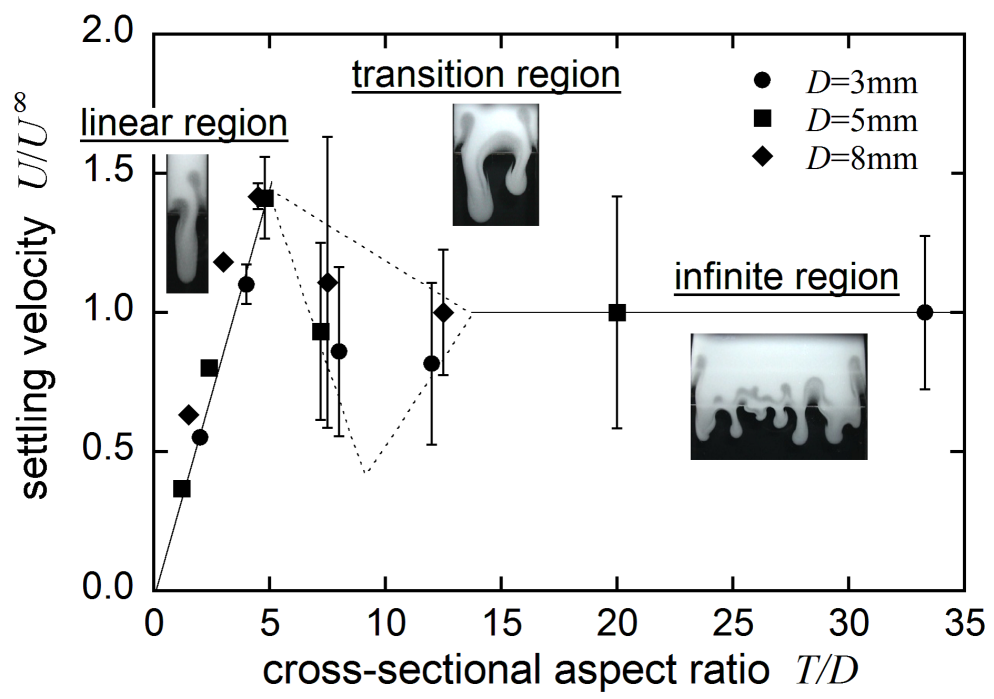

Fig.5 Settling velocity of particulate suspension in rectangular channel (reconstruction of Fig.8 in Harada et al. [10]) 


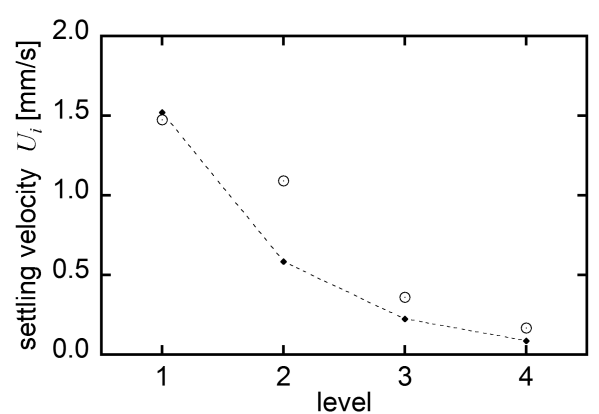

(a) $\phi=0.03$

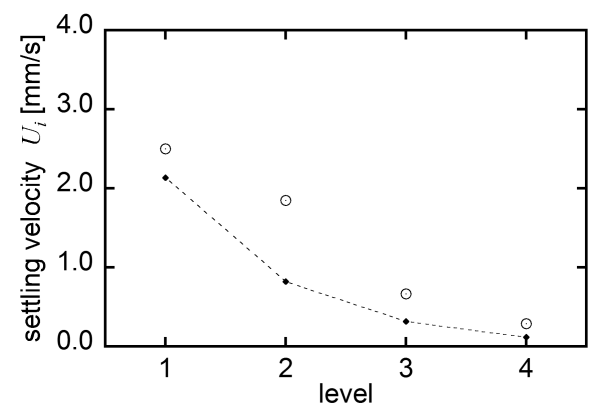

(b) $\phi=0.05$

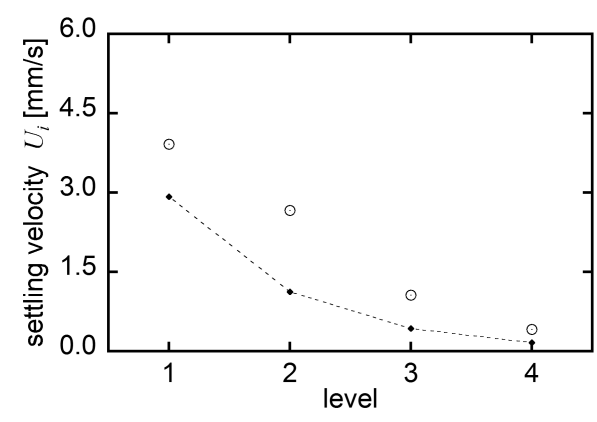

(c) $\phi=0.08$

Fig.6 Settling velocity of glass particles for each level of fractal channel (open circles: experimental results, dotted lines: prediction from Eq.(6)) 


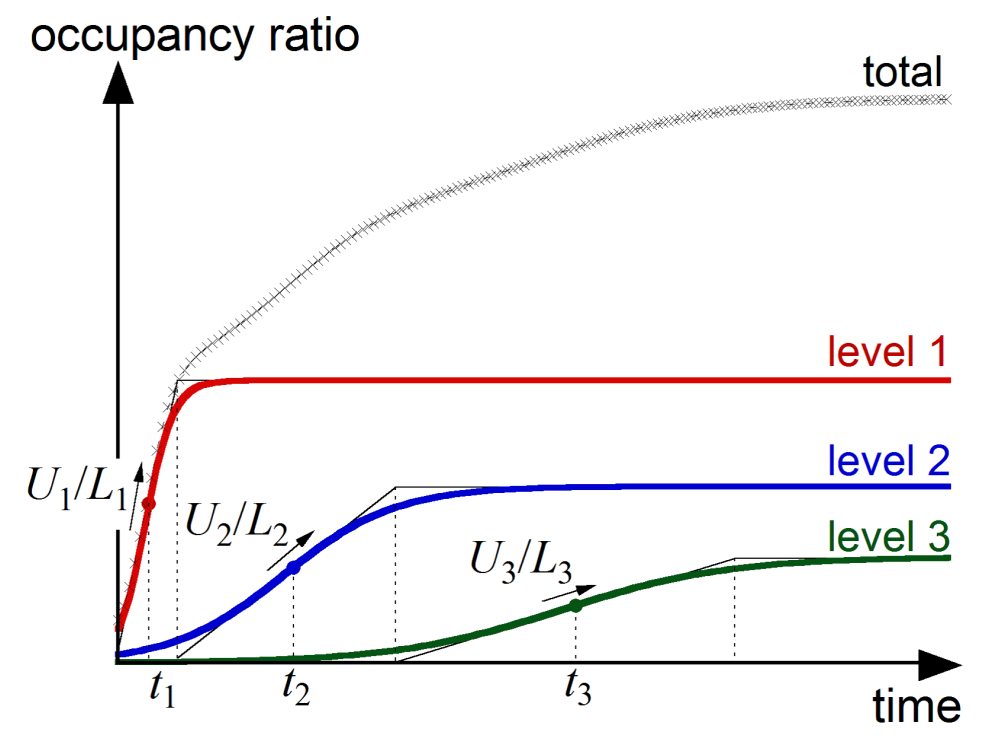

Fig.7 Conceptual diagram of model for invasion of particulate suspension into fractal channel 


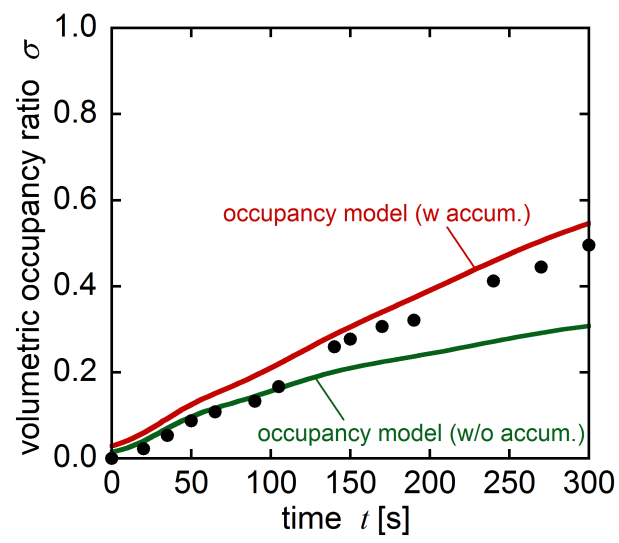

(a) $\phi=0.03$

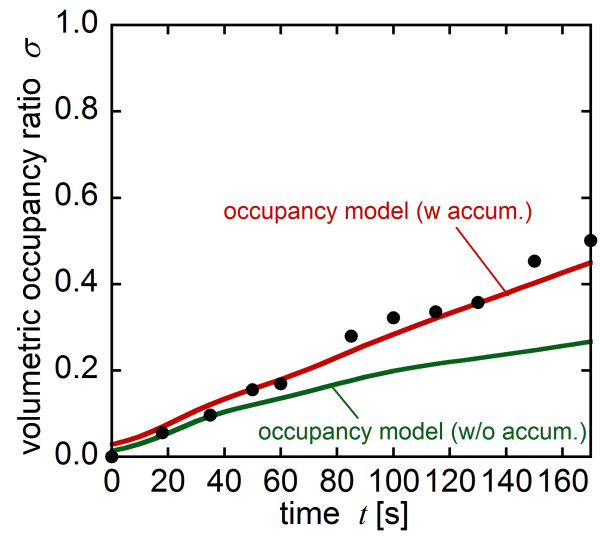

(b) $\phi=0.05$

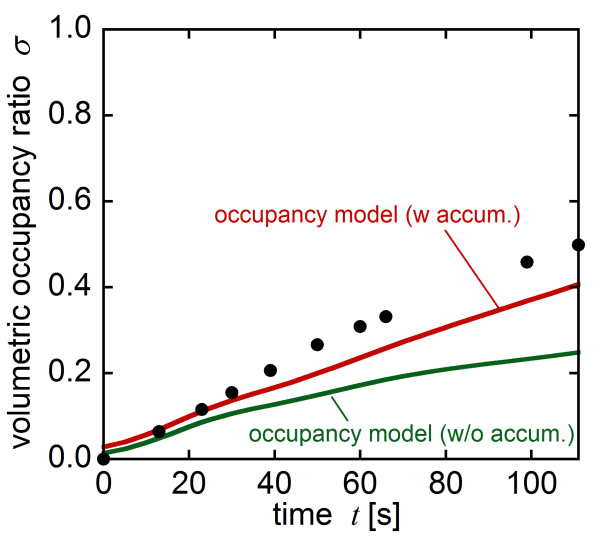

(c) $\phi=0.08$

Fig.8 Comparison between experimental and model results for volumetric occupancy ratio 